\title{
Sterile Root Canal: Obturation is Not Sterilization
}

\author{
RS Carlson* \\ University of Michigan School of Dentistry, USA \\ *Corresponding author: RS Carlson, University of Michigan School of Dentistry, USA \\ To Cite This Article: RS Carlson. Sterile Root Canal: Obturation is Not Sterilization. Am J Biomed Sci \& Res. 2019 - 1(3). AJBSR.MS.ID.000528. \\ DOI: 10.34297/AJBSR.2019.01.000528
}

Received: February 06, 2019 | Published: February 22, 2019

\begin{abstract}
The case is simple. Mixed distinctions beget sloppy science and practice. Grossman stated in 1972 that "root canals can and need to be sterilized" that "...they must be sterilized" [1]. This dictum and advice for all has been sidestepped in the past Century. We now have "disinfection" and "obturation" as surrogates for successful sterile root canal therapy.
\end{abstract}

My hypothesis is successful RTC (Root Canal Therapy) in any event is not possible without sterilization." I am sure to have opposition, vehement challenges...and more drivel. Show me a sterile root canal and I'll show you success!

Define sterile

"Sterility can be defined as the freedom from the presence of viable microorganisms."

Further, Sterile or Sterility refers to any process that eliminates or kills all forms of life from an item or field...an inability of a living organism to reproduce.

\section{Define disinfection}

Disinfectants are antimicrobial agents that are applied to the surface of non-living objects to destroy microorganisms that are living on the objects.

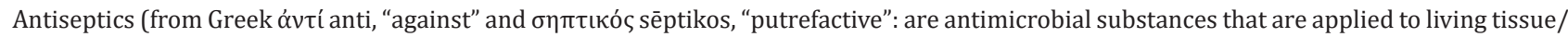
skin to reduce the possibility of infection, sepsis,

or putrefaction.

\section{Define Obturation}

(canal obturation) filling of a root canal completely and densely with a nonirritating hermetic sealing agent. Further, canal obturation in root canal therapy, the filling of the pulp, or root, canal completely and densely with a nonirritating hermetic sealing agent. Also called root canal filling. See also root canal therapy. Mosby's Medical Dictionary, $9^{\text {th }}$ edition.

Understand that the tissues below the skin of a human, the subcutaneous tissues, dwell as a "sterile" space. This sterility is essential for

\section{Introduction}

In normal healthy metabolism we do not have inflammation due to micro-organisms, since Inflammation is an adaptive process to the noxious stimuli that the human body is constantly exposed to. From the local inflammatory response to a full-blown systemic inflammation, a wide complex sequence of events occurs.

Persistent immunosuppression and catabolism may ensue, until multiple organ failure finally sets in... A thorough understanding of these events (how they begin, how they evolve, and how to modulate them) is imperative, but yet poorly studied. This review aims to consolidate current knowledge of these events so that the management of these patients is not only evidence-based, but also built on an understanding of the inner workings of the human body in health and in disease [2].

Our pernicious collapse of distinctions as being distinct has taken hold of the profession and therefore the Public creating havoc in health care.

The ill effects on human beings with root canals being done without sterility is enormous. Here are some other comments regarding the issues related to metabolism: 
"Sites of ongoing inflammation and triggered immune responses are characterized by significant changes in metabolic activity. Recent studies have indicated that such shifts in tissue metabolism result from a combination of profound recruitment of inflammatory cells (neutrophils and monocytes) and high proliferation rates among lymphocyte populations [3].

The resultant shifts in energy supply and demand can result in metabolic acidosis and diminished delivery and/or availability of oxygen, leading to hypoxia extensive enough to trigger transcriptional and translation changes in tissue phenotype. Such phenotypic shifts can imprint fundamental changes to tissue metabolism..." [3].

"The dynamic interplay of leukocytes and parenchymal cells during disease defines an elegant lesson in biology. In particular, studies of model disease systems have allowed for the identification of metabolomic changes now well accepted in the scientific literature. The discovery of differences and similarities...... will continue to teach us important lessons about the complexity of biological systems" [3].

We can see, if we will to see, that the distinctions disinfection, antispetics, obturation are not even close to sterilization and that is our dilemma in the profession. No one has addressed this issue, but I do here, in this document. Our colleagues insist that they can sterilize and obturate the radiculary anatomy of the canals of Odontons. I challenge them to obturate and sterilize the one we see in (Figure 1). This is a photo micro- graph from" EndoCal 10 Obturation Voids in Root Canal and Isthmus of a Human Premolar: A Synchrotron micro-CT Imaging Study [4].

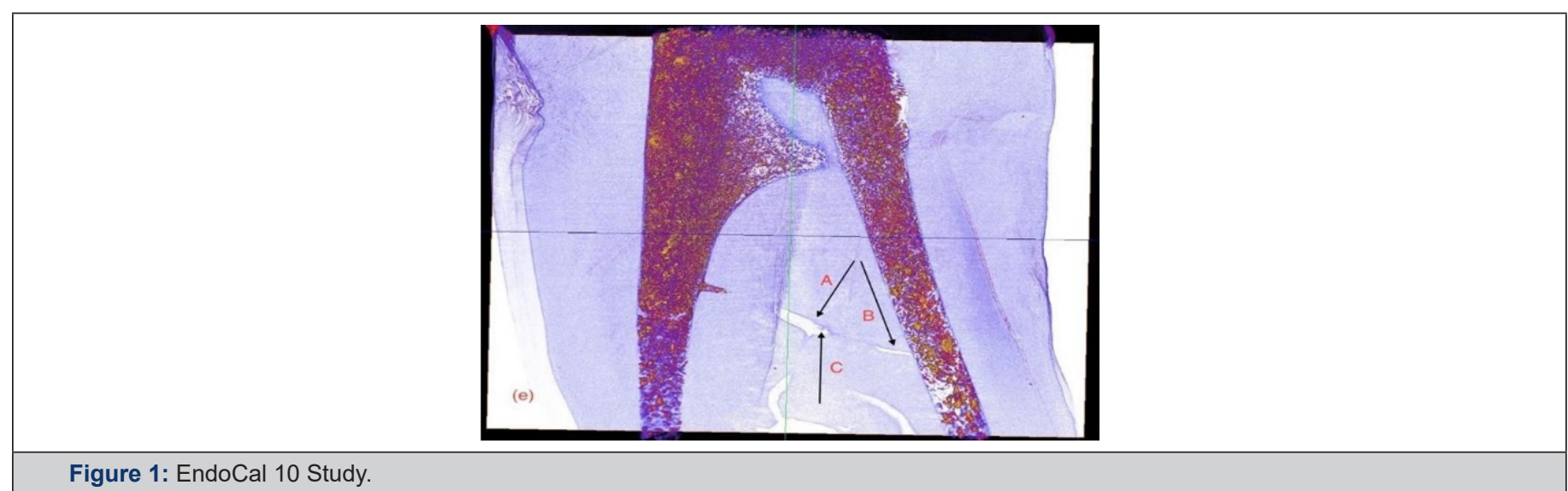

The voids seen at $\mathrm{A}, \mathrm{B}$, and $\mathrm{C}$ are only of a fraction of the accessory canals in this premolar, not to mention the billion and billions of dental tubules that transect the sterile root structure from pulp chamber to peripheral cementum/periodontium/bone hematopoetic tissues. The presence of pulp tissue is still seen in C. Micro- organisms were not retrieved in this study, but neither was there an attempt to retrieve. The purpose of the study was the obturation effectiveness of EndoCal 10, which they concluded was as good as gutta-percha. (Ho-Hum!) At the level of about 3 microns in diameter the canals in human Odontons are superhighways or vast oceans to fill with sealants used in root canal therapy. And we find huge voids in the filling material of Root Canal systems as well as voids in the canals that and not able to be penetrated with sealants.

The study on obturation concluded the following regarding (Figure 2), the isthmus between the premolars two roots, left and right:

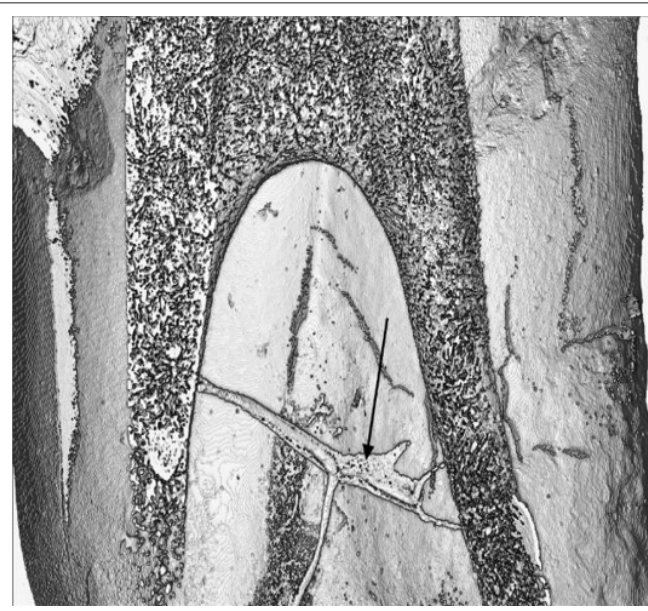

Figure 2: EndoCal 10 Study. 
a. "The isthmus (see arrow Figure 2) is a continuous one connecting both root canals, and accordingly can be classified as a type V isthmus as defined in the literature (Hsu \& Kim 1998).

b. Some 2D and 3D images showed remnants of pulp tissue inside the isthmus.

c. The isthmus was partially obturated and Endocal 10 was able to flow to a certain extent from one end as shown in the series of images in Figure 2

d. This is an indication that no hard debris resulting from the preparation of the root canals plugged the isthmus.

e. Through 3D visualization, we observed that the geometry of the isthmus is tubular in structure. It is narrow at the root canal connecting ends and has dead-end branches emerging from it. The isthmus' diameter increases to a maximum in between its two ends, which causes a pressure drop in the Endocal's flow through it. The pressure drop is an impeding factor to the flow of Endocal 10 through the isthmus.

f. As shown in (Figures $1 \& 2$ ) the isthmus is slanted downwards from the left root canal to the right one. Accordingly, it was easier for the EndoCal to flow downwards and partially obturate the isthmus at the left end, rather than flow upwards at the right end."

The results of this particular study were stated as thus:

"We used Avizo 9.0 for 3D visualization of the premolar and observed three distinct features in the images: a vertical fracture along the side of one of the roots, voids within the obturated pulp chamber and apex of the nonfractured root, and partial obturation within the isthmus between the two root canals. Since we have already prepared a manuscript identifying and analyzing the cause of the vertical fracture along one side of one of the roots, the latter two observations are addressed in this manuscript."

\section{Conclusions}

"Similar to other obturating materials used in endodontic therapy, voids may appear in parts of the pulp chamber, apical region of the root canal, and isthmus following the application of EndoCal 10.

As illustrated through 3D rendering, the root canal isthmus in this study is characterized by a non-uniform diameter, branches, and tissue within it. With conventional endodontic therapy, it is not possible to completely obturate an isthmus with a structure similar to the one visualized in this study. This is attributed to its varying diameter, its branching architecture, and the tissue remaining in it. Through 3D visualization of the isthmus between the two root canals in this study, we categorize this isthmus as a type V.

Again, I state that this presentation on EndoCal 10, although brilliantly done, is speaking only about root canal obturation and mentions nothing about "sterility" or the need to "sterilize." It seems that they have glossed over a vitally critical and important key point in any successful equation for root canal therapy—sterility!

My realization at this time is that ancillary debate on disinfecting, antisepting, obturating to success in Root Canal Therapy is impracticable, impossible at this time. Without clear "sterilization of the canal system" one will meet with immediate failures and slow failures, in all cases, due to many factors presented in current research. My paper you read only speaks to an essential factor in success, "sterility," not mentioning the, genotoxicity, cytotoxicity, mutagenicity, and endotoxemia associated with RCT fillers that is not addressed adequately in sloppy science, or at all.

\section{References}

1. Grossman L (1972) Sterilization of Infected Root Canals The Journal of the American Dental Association. 85(4): 900-905.

2. Varela ML, Mogildea M, Moreno I, Lopes A (2018) Acute Inflammation and Metabolism. Inflammation 41(4): 1115-1127.

3. Ford LB, Cerovic V, Milling SW, Graham GJ, Hansell CA (2014) Characterization of conventional and atypical receptors for the chemokine CCL2 on mouse leukocytes. J Immunol 193(1): 400-411.

4. In Proceedings of the $11^{\text {th }}$ International Joint Conference on Biomedical Engineering Systems and Technologies (BIOSTEC 2018). Volume 2. Bioimaging, p. 43-49. 